\title{
Design of 1553B Protocol for Improving the Fault Diagnosability
}

\author{
Shuai Feng ${ }^{1, *}$, Yingfa Zhang ${ }^{1}$, Mingchao Song ${ }^{1}$ and Biao Cai ${ }^{1}$ \\ ${ }^{1}$ Beijing Institute of Control Engineering, Beijing 100190, China \\ ${ }^{*}$ Corresponding author
}

\begin{abstract}
B system is described and an application layer communication protocol based on 1553B is introduced in this paper. The protocol adopts the 'broadcast-and-echo' strategy, which can provide lots of fault information of the system in many ways to improve the ability of fault diagnosis. The validity of the protocol is proved by analysis and experiments. The proposed protocol is practical with low cost, and has been applied to the GNC system of a spacecraft.
\end{abstract}

Keywords-1553B; application layer protocol; fault diagnosis

\section{INTRODUCTION}

1553B bus has been widely used in various systems [1], especially in the key systems, such as attitude control system, life support system and so on. However, the harsh factors in the environment, for example, high/low temperature, electrostatic impulse and single particle damage, are likely to cause the failure of 1553B system. Due to its criticality, failure to detect and deal with faults in time is likely to cause serious consequences, such as the loss of astronauts and the failure of the mission. So the rapid fault handling is important for 1553B system.

In order to deal with the system fault, enough abnormal information should be detected first to locate the failure. Therefore, during the designing of the system, the fault diagnosability must be taken into account, which means that different faults should be fully characterized by different abnormal phenomenon [2]. So that when an abnormal phenomenon occurs, the fault can be quickly located.

Although there are a few of studies on the fault diagnosis of 1553B system [3, 4, 5], most scholars adopt the off-line expert system, fuzzy logic [3] and other probabilistic diagnosis, which cannot provide accurate result. And the fault diagnosis algorithms are mostly carried out after the system design is completed, and use little information of the actual system, so that these algorithms cannot provide much help in the actual application.

In order to improve the fault diagnosability, this paper designs an application layer communication protocol based on 1553B during the design process of the GNC (guide, navigation and control) system of a spacecraft. The protocol adopts the 'broadcast-and-echo' strategy, which can provide lots of abnormal information of the system in many ways for fault positioning to improve the ability of fault diagnosis.
This paper introduces the basic knowledge of 1553B system, and describes the proposed protocol, then shows how to use the abnormal phenomenon of the communication to diagnose the fault by analysis and experiments, and summarizes the work in the last.

\section{1553B SYSTEM}

$1553 \mathrm{~B}$ bus is a master-slave, time-division multiplexed serial data bus. The date rate is $1 \mathrm{MHz}$, and data is encoded as the Manchester II dual-phase code.

\section{A. Hardware Components}

A typical diagram of 1553B system is shown in Figure I. The 1553B system consists of a main bus cable (redundant bus cables are allowed), a number of terminals and terminating resistors. The detailed 1553B hardware specification is listed in [6].

There are three types of terminals: BC (Bus Controller), RT (Remote Terminal) and BM (Bus Monitor). BC is the bus master, and it controls all the data transmission. BM monitors and records the data flowing in the bus. $\mathrm{RT}$ sends data to $\mathrm{BC}$ or receives data from $\mathrm{BC}$ under the control of $\mathrm{BC}$.

RT typically consists of a protocol controller (also called interface chip), a processor and a subsystem. The subsystem typically is a sensor or an actuator, which provides the measured data or executes the instruction. The processor processes the data, and the protocol controller usually is a complete integrated interface between the processor and the 1553B bus.

\section{B. 1553B Protocol}

1553B protocol can be divided into three layers [7]: the physical layer, the transport layer and the application layer, as shown in Figure II.

The physical layer protocol and the transport layer protocol are defined specifically in 1553B standard, while the user needs to design the application layer protocol.

The data bit is encoded as the $1 \mathrm{MHz}$ Manchester II dualphase code in the physical layer.

In the transport layer, only there types of words are supported: command word, data word and status word. And they compose 10 kinds of message formats, including 6 non- 
broadcast formats and 4 broadcast formats. The detailed specification of the formats is described in [6].

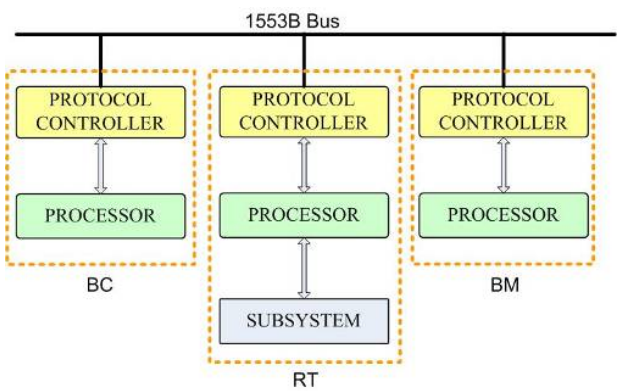

FIGURE I. 1553B SYSTEM COMPONENTS

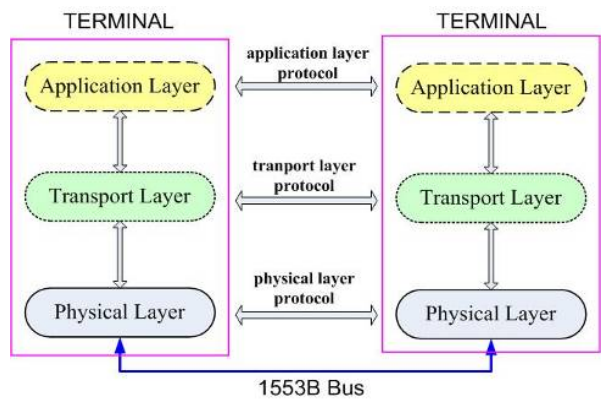

FIGURE II. THREE LAYERS OF 1553B PROTOCOL

In the application layer protocol, designed in this paper, two message formats are mainly used: broadcasted mode command, and non-broadcast RT to BC transfer, which are shown in Figure III.

\section{THE APPLICATION LAYER PROTOCOL}

The following 1553B application layer protocol is designed for 1553B system as shown in Figure I.

\section{A. Timing}

1) The BC takes the following operation with a cycle.

- At the beginning of the cycle, a mode command with data word is broadcasted to all the RT on the bus.

- After a specific time (used for RT to prepare the data), $B C$ requests the data from $\mathrm{RT}$.

- The received data is processed and the control instruction is send to RT.

2) The RT operates as following

- After receiving the broadcasted command from BC, RT collects data from the subsystem and writes the data to the protocol controller (also called the interface chip).

- Under BC's request, RT protocol controller transfers its data to $\mathrm{BC}$ without the control of the RT processor.

- $\quad$ RT executes the control instruction when receiving it.

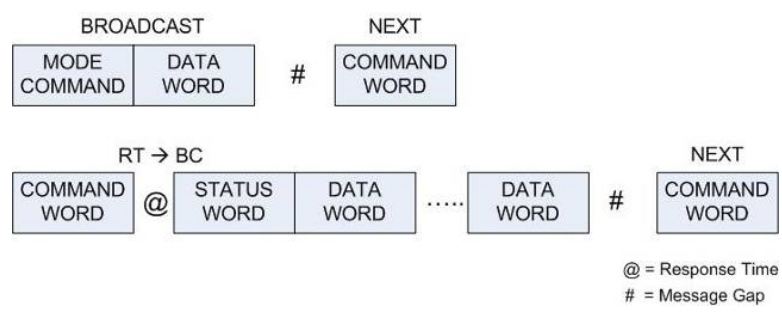

FIGURE III. TWO 1553B MESSAGE FORMATS

The format of broadcasted mode command with data word, shown in Figure III, is defined as follows.

- $\quad$ The mode command word is fixed as 0xFBF1.

- The data word is a counter, and it increases every cycle.

The broadcasting is quite useful in several aspects.

- As the heartbeat of the 1553B system.

- To ensure all the RT sample and prepare the data at the same time.

- To diagnose the RT working state (that will be explained in the next section).

2) RT data

In the protocol, though different RT generates different data, the last three words of all the RT data are defined the same as shown in Table I.

The counter send back by RT should be the same with the counter BC just broadcasted. That is the reason why the protocol strategy is called 'broadcast-and-echo'.

\section{ANALYSIS AND EXPERIMENTS}

\section{A. Analysis}

Using the protocol, several types of system faults can be diagnosed from the abnormal communication phenomenon monitored by BM as shown in Table II.

The RT interface chip may respond to the request of BC by itself as long as it is initialized correctly by processor. So in some situation, when the processor can't work, the RT can still send out data or receive data, though the data is not updated by processor.

The difference of the counter send back by RT with the counter broadcasted by BC simply means that the RT data has not been updated correctly.

\section{B. Experiments}

The protocol introduced above is tested in the GNC (guide, navigation and control) system of a spacecraft. Figure IV shows the scene of system during experiments.

\section{B. Message Format}

1) BC data 


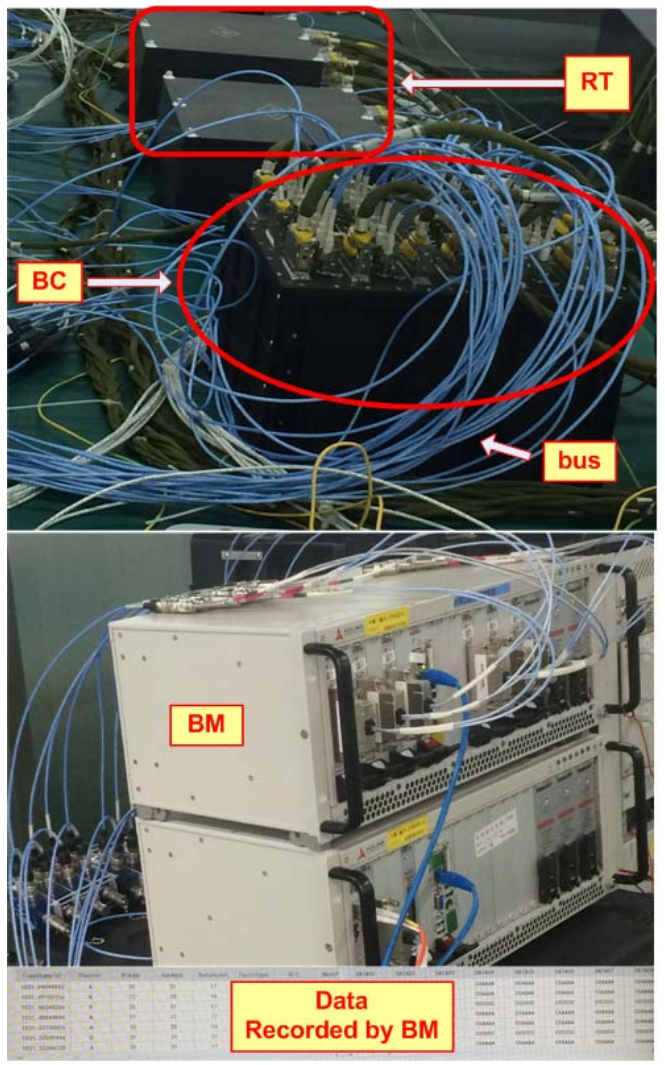

FIGURE IV. THE SCENE OF EXPERIMENTS

In the experiments, mainly two RT terminal faults are created to test the fault diagnosability of the system with the protocol.

\section{1) Processor fault}

TABLE I. RT DATE FORMAT

\begin{tabular}{|l|l|}
\hline \multicolumn{1}{|c|}{ Word Index } & \multicolumn{1}{c|}{ Definition } \\
\hline The first to $(\mathrm{N}-3)^{\text {th }}$ words & Not defined \\
\hline The $(\mathrm{N}-2)^{\text {th }}$ word & The health flag of the subsystem \\
\hline The $(\mathrm{N}-1)^{\text {th }}$ word & The counter just broadcasted by BC \\
\hline The $\mathrm{N}^{\text {th }}$ (last) word & The sum of all the above data \\
\hline
\end{tabular}

TABLE II. FAULT DIAGNOSIS

\begin{tabular}{|l|l|l|}
\hline \multicolumn{1}{|c|}{ Abnormal phenomenon } & \multicolumn{1}{|c|}{$\begin{array}{c}\text { Fault } \\
\text { diagnosed }\end{array}$} \\
\hline 1 & There is no broadcast data in the bus & BC faults \\
\hline 2 & RT dose not respond to BC's request & $\begin{array}{l}\text { RT interface } \\
\text { chip faults }\end{array}$ \\
\hline 3 & RT responds to BC's request wrongly & $\begin{array}{l}\text { RT interface } \\
\text { chip faults }\end{array}$ \\
\hline 4 & The sum in the RT data is not correct & $\begin{array}{l}\text { RT processor } \\
\text { faults }\end{array}$ \\
\hline 5 & $\begin{array}{l}\text { The counter send back by RT is different } \\
\text { from the counter broadcasted by BC }\end{array}$ & $\begin{array}{l}\text { RT processor } \\
\text { faults }\end{array}$ \\
\hline 6 & $\begin{array}{l}\text { The subsystem health flag shows wrong } \\
\text { state }\end{array}$ & $\begin{array}{l}\text { RT subsystem } \\
\text { faults }\end{array}$ \\
\hline
\end{tabular}

The fault is generated by running special software to make the processor keep on restarting itself in several seconds.

The data in Figure V, recorded by BM, shows the abnormal communication phenomenon of the processor fault.

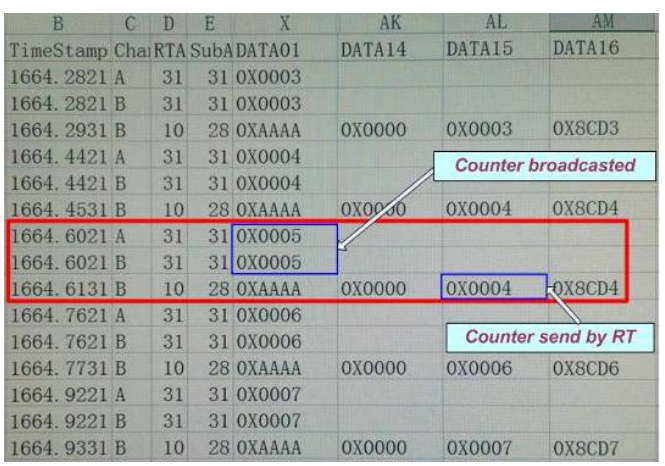

FIGURE V. ABNORMAL DATA

From the data, it is easy to see that the counter send back by $\mathrm{RT}$ is not the same with the counter broadcasted by BC. This unusual appearance means that the processor is not working well and the data it sends out is not updated, even though the $\mathrm{BC}$ still can get the old data from RT.

2) Protocol controller fault

The fault is created by loosing the chip's contact with the circuit board. In this case, the RT could not respond to the BC's data request simply.

These analysis and experiment results above show that different faults can be fully characterized by different abnormal phenomenon and that the fault diagnosability of the system has been improved effectively.

\section{CONCLUSION}

This paper analyzed the general structure of 1553B system, and designed an application layer communication protocol based on 1553B to improve the fault diagnosability. The protocol adopts the 'broadcast-and-echo' strategy, which means that BC sends a counter to RT and RT sends the counter back to BC along with its own data. Using this strategy, the abnormal phenomenon of communication can provide lots of fault information of the system in many ways. 
The analysis and experiment result shows the validity of the protocol. And the proposed protocol has been applied to the GNC system of a spacecraft.

\section{REFERENCES}

[1] L. Guoxing, L. Ming, and H. Ruchang, "Way of real-time acquisition 1553B Data in Flight Test”, Computer Measurement and Control. China, vol. 24, pp.120-121, 2016.

[2] H. Yongzhao, L. Qingdong, R. Zhang, and L. Chengrui, "Overview of fault diagnosability evaluation methods for contnuous systems”, Control and Decision. China, vol. 31, pp. 2113-2121, December 2016.

[3] S. Yahui, and Z. Yifan, "Fault diagnosis of 1553B bus system in missile by fuzzy petri net”, Computer Measurement and Control. China, vol. 21, pp. 1251-1253, 2013.

[4] K. Qingchun, X. Mingqing, and Q. Xiaolin, "Design of avionic fault diagnosis expert system based on 1553B bus”, Journal of Gun Launch and Control. China, vol. 3, pp. 68-72, 2009.

[5] J. Minli, and Z. Xuemin, "Fault diagnosis of 1553B bus network using weighted fuzzy petri net”, Computer Measure and Control. China, vol. 21, pp. 2056-2058, 2013.

[6] MIL-STD-1553 Designer’s Guide. Data Device Corporation, 2003.

[7] C. Liang, L. Lixia, and L. Jingyu, "Degign and Analysis of Information Flow in Missile Control System Based on 1553B Protocol”, Computer Measurement and Control. China, vol. 20, pp.2180-2182, 2012. 